\title{
A comparative study of Ligophorus uruguayense and L. saladensis (Monogenea: Ancyrocephalidae) from Mugil liza (Teleostei: Mugilidae) in southern Brazil
}

\author{
Natalia C. Marchiori ${ }^{1}$, Antoine Pariselle ${ }^{2,3}$, Joaber Pereira Jr. $^{4}$, Jean-François Agnèse ${ }^{2}$, Jean-Dominique Durand ${ }^{5}$ \\ and Maarten P.M. Vanhove $6,7,8,9$
}

\author{
${ }^{1}$ Empresa de Pesquisa Agropecuária e Extensão Rural de Santa Catarina (Epagri), Campo Experimental de Piscicultura de Camboriú, \\ Camboriú, Santa Catarina, Brasil; \\ ${ }^{2}$ Institut des Sciences de l'Évolution, IRD-CNRS-Université Montpellier 2, Montpellier, France; \\ ${ }^{3}$ Present address: Institut de Recherche pour le Développement (IRD), Yaoundé, Cameroon; \\ ${ }^{4}$ Laboratory of Parasite Biology of Aquatic Organisms, Oceanographic Institute, Federal University of Rio Grande Foundation, Rio \\ Grande, Rio Grande do Sul, Brazil; \\ ${ }^{5}$ Institut de Recherche pour le Développement (IRD), University of Montpellier 2, Montpellier, France; \\ ${ }^{6}$ Laboratory of Biodiversity and Evolutionary Genomics, Department of Biology, University of Leuven, Leuven, Belgium; \\ ${ }^{7}$ Department of Botany and Zoology, Faculty of Science, Masaryk University, Brno, Czech Republic; \\ ${ }^{8}$ Invertebrates Unit, Biology Department, Royal Museum for Central Africa, Tervuren, Belgium; \\ ${ }^{9}$ Institute of Marine Biological Resources and Inland Waters, Hellenic Centre for Marine Research, Anavyssos, Greece
}

\begin{abstract}
Representatives of Ligophorus Euzet et Suriano, 1977 were found on the gills of Mugil liza Valenciennes caught in southern Brazil. They were identified as Ligophorus uruguayense Failla Siquier et Ostrowski de Núñez, 2009 and Ligophorus saladensis Marcotegui et Martorelli, 2009, even though-specific identification proved to be difficult due to inconsistencies in some diagnostic features reported for these two species. Therefore, a combined morphological and molecular approach was used to critically review the validity of these species, by means of phase contrast and confocal fluorescence microscopical examination of sclerotised hard parts, and assessing the genetic divergence between $L$. saladensis, L. uruguayense and their congeners using rDNA sequences. The main morphological differences between the two species relate to the shape of the accessory piece of the penis and the median process of the ventral bar. The accessory piece in L. uruguayense is shorter than in L. saladensis, has a cylindrical, convex upper lobe and straight lower lobe (vs with the distal tip of the lower lobe turning away from the upper lobe in the latter species). The ventral bar has a V-shaped anterior median part in L. uruguayense ( $v$ s U-shaped in L. saladensis). The two species are suggested to be part of a species complex together with L. mediterraneus Sarabeev, Balbuena et Euzet, 2005. We recommend to generalise such comparative assessment of species of Ligophorus for a reliable picture of the diversity and diversification mechanisms within the genus, and to make full use of its potential as an additional marker for mullet taxonomy and systematics.
\end{abstract}

Keywords: morphology, molecular systematics, mullet, parasite, taxonomy

The identification of species belonging to Ligophorus Euzet et Suriano, 1977, monogenean gill parasites of mullets (Mugilidae), mainly relies on the morphology and size of the sclerotised parts of the haptor and of the male copulatory complex (Blasco-Costa et al. 2012). However, distinguishing between closely-related species may be very difficult, not only because of the small size of these structures, but also because of their close resemblance under optical microscopy. Common flaws in describing species of Ligophorus include inadequate flattening of the haptoral parts of fixed specimens and considering the differences between dorsal and ventral views of the ventral bar as intraspecific or interspecific character variation (Dmitrieva et al. 2009a and references therein). Overlooking functional aspects of the structures used for morphological identification has also led to errors (Dmitrieva et al. 2009b). Moreover, the relationship between morphology-based and molecular taxonomy is not yet clear, although congruence between the two approaches was reported to be high by Sarabeev and Desdevises (2014). 
To date, six species of Ligophorus were described from Mugil liza Valenciennes (syn. Mugil platanus Günther see Fraga et al. 2007) in South America: Ligophorus uruguayense Failla Siquier et Ostrowski de Núñez, 2009 from Laguna de Rocha, Uruguay, Ligophorus saladensis Marcotegui et Martorelli, 2009 from Samborombón Bay, Argentina and four other species from the Guandu River, state of Rio de Janeiro, Brazil, namely L. brasiliensis, L. guanduensis, L. lizae and L. tainhae, all described by Abdallah et al. (2009), i.e. Abdallah, Azevedo et Luque, 2009.

Specimens of M. liza from southern Brazil were sampled for investigation of their monogenean fauna. Numerous individuals of Ligophorus were found on the gills of this fish host. They were identified as L. uruguayense and L. saladensis, even though specific identification proved to be difficult mostly because some of the diagnostic morphological characters reported for these species (e.g. presence/ absence of transverse annulations at the distal end of the vaginal tube as well as the presence of a thick process at the distal end of the inner root of ventral anchors) seemed to vary continuously in the sample. In addition, the male copulatory complex of these two closely-related species may be very similar, depending on the position in which this structure is observed. Therefore, the validity of both species needed to be critically reviewed. In a recent revision, Sarabeev et al. (2013) indicated problems in the original descriptions of these species, such as a surprisingly wide range in haptoral morphometrics for L. uruguayense and recommended to revise the morphology of the reproductive organs for both species.

The present study critically reviews the validity of L. uruguayense and L. saladensis using an integrated approach combining both morphological and molecular analyses.

\section{MATERIALS AND METHODS}

In October 2012, thirty fingerlings of Mugil liza (total length of $3.0 \pm 0.4 \mathrm{~cm}$; weight of $0.3 \pm 0.9 \mathrm{~g}$ ) were collected from a stream that flows into the Atlantic Ocean at Querência, Cassino Beach, Rio Grande do Sul, Brazil (32 $\left.{ }^{\circ} 11^{\prime} \mathrm{S} ; 52^{\circ} 10^{\prime} \mathrm{W}\right)$. For each fish specimen, gills were excised, fixed in $95 \%$ ethanol and kept separately from the rest of the body, which was then also fixed in 95\% ethanol and kept in another glass jar.

Monogeneans were collected from the fish gills and some of them were mounted in Hoyer's medium (Humason 1979) on a slide to study the morphology of the sclerotised hard parts using a Reichert-Jung Polyvar compound microscope at a magnification of $\times 1000$ using interference phase contrast.

Specimens found in this host were identified as Ligophorus uruguayense and L. saladensis, both originally described from M. liza in Uruguay and Argentina, respectively (Failla Siquier and Ostrowski de Núñez 2009, Marcotegui and Martorelli 2009). Both species are very similar to each other, but could be most reliably distinguished on the basis of the shape of the accessory piece of the male copulatory complex (see Failla Siquier and Ostrowski de Núñez 2009, Marcotegui and Martorelli 2009). The specimens that could not be identified to the species level reliably were excluded from the morphometrical study. To confirm the validity of both species, specimens that could be unquestionably assigned to one of the species were used for molecular study.

Measurements of the sclerotised structures in 18 specimens of L. uruguayense and 10 of L. saladensis (except for vagina length, which was measured in one and three specimens, respectively) were taken as defined by Failla Siquier and Ostrowski de Núñez (2009) using a Leica DM2500 microscope and LAS 6.3 software. They are given in micrometres $(\mu \mathrm{m})$, with the range followed by the mean in parentheses. The following abbreviations for the characters are used throughout the text: DAA - dorsal anchor total length; DAB - dorsal anchor main part length; DAC - dorsal anchor outer root length; DAD - dorsal anchor inner root length; DDIOR - distance between inner and outer root of dorsal anchors; LWL - penis accessory piece lower lobe length; PAPL - penis accessory piece total length; PL - total length of penis; VAC ventral anchor outer root length; VAD - ventral anchor inner root length; VBAP - distance between membranous anterior processes of ventral bar; VBL - ventral bar length; VDIOR - distance between inner and outer root of ventral anchors. Voucher specimens were deposited in the Helminthological Collection of the Instituto Oswaldo Cruz (CHIOC), Rio de Janeiro, Brazil (CHIOC 37951a, $37951 b-$ L. uruguayense and 37952a, 37952b - L. saladensis).

For molecular identification, other specimens were mounted in Malmberg's medium (ammonium picrate-glycerine - Malmberg 1957) and analysed under a phase contrast microscope. Only specimens with a well-characterised male copulatory complex (MCC) (clearly a usable character based on the original descriptions) were considered as identified to the species level: this selection took into consideration the observation of the shape of the upper and lower lobes of this structure, which was greatly facilitated by the use of Malmberg's medium. Specimens were then photographed and later each individual was dismounted and rinsed over a 24 -hour period with $95 \%$ ethanol. After that, they were transferred, under a stereomicroscope, into a $2 \mathrm{ml}$ Eppen$\operatorname{dorf}^{\circledR}$ tube filled with $15 \mu \mathrm{l}$ of sterilised nuclease-free distilled water, and grinded with the aid of a disposable micropipette tip (used for P20-200 micro-pipettor).

DNA suspended in water with lipids, proteins and other components was directly used as template for amplification. DNA concentration, measured with a Qubit (Life Technologies, Carlsbad, CA, USA) fluorometer with a Qubit (Life Technologies) dsDNA BR assay Kit, varied between $0.066 \mu \mathrm{g} / \mu \mathrm{l}$ and $0.120 \mu \mathrm{g} / \mu \mathrm{l}$. Primer combinations followed Blasco-Costa et al. (2012). A portion (D1-D2) of 28S rDNA was amplified using U178 (5'-GCACCCGCTGAAYTTAAG-3') (Lockyer et al. 2003) and LSU1200R (5'-GCATAGTTCACCATCTTTCGG-3') (Littlewood et al. 2000). For the ITS-1 rDNA region, Lig18endF (5'-GTCTTGCGGTTCACGCTGCT-3') and Lig5.8R (5'-GATACTCGAGCCGAGTGATCC-3') (Blasco-Costa et al. 2012) were used. The amplification protocol consisted of 40 cycles beginning with $2 \mathrm{~min}$ at $93{ }^{\circ} \mathrm{C}$ for initial denaturation followed by cycles of $30 \mathrm{~s}$ at $93^{\circ} \mathrm{C}, 30 \mathrm{~s}$ at $56^{\circ} \mathrm{C}$ for annealing, $1 \mathrm{~min} 30 \mathrm{~s}$ at $72^{\circ} \mathrm{C}$ for extension, with a final 5 min extension step at $72^{\circ} \mathrm{C}$. The different reagents' final concentrations were as followed: GoTaq Flexibuffer (Promega) $1 \times, \mathrm{MgCl}_{2} 2.5 \mathrm{mM}$, PCR nucleotide mix, $0.2 \mathrm{nM}$ of each DNTP, forward and reverse primers $1 \mu \mathrm{M}$ each, 2 U GoTaq (Promega) DNA polymerase, template DNA $0.2 \mu \mathrm{g}$ (between 1.6 to $3 \mu 1$ depending on the DNA extract concentration), and nuclease-free water to a total volume of $20 \mu 1$. 


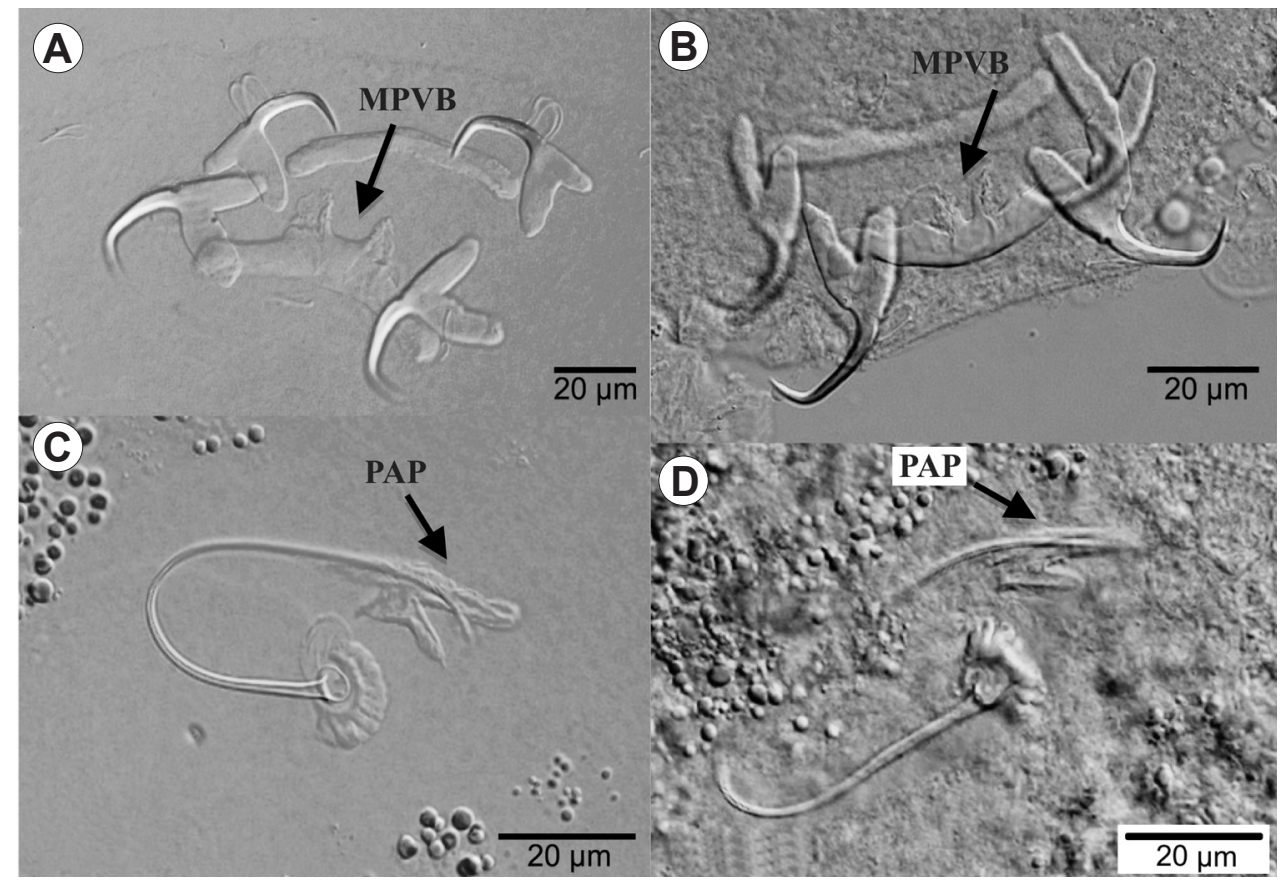

Fig. 1. Photomicrographs of hard parts of Ligophorus saladensis Marcotegui et Martorelli, 2009 (A, C) and Ligophorus uruguayense Failla Siquier et Ostrowski de Núñez, 2009 (B, D), both from Mugil liza Valenciennes in southern Brazil. A, B - haptor; C, D - male compulatory complex. Abbreviations: MPVB - median process of the ventral bar; PAP - penis accessory piece.

Sequences were aligned using Clustal W (Thompson et al. 1994) implemented in MEGA v.6 (Tamura et al. 2013). The latter software was also used to calculate the genetic distances and for model selection and phylogenetic tree building. With this phylogeny reconstruction, we do not aim to redo or repeat the work by Blasco-Costa et al. (2012) or Sarabeev and Desdevises (2014); it is intended merely to situate L. saladensis and L. uruguayense among their congeners that have been genetically characterised. Following Blasco-Costa et al. (2012), both fragments are analysed separately. Based on the Bayesian Information Criterion, the optimal model for molecular evolution was the Kimura two parameter (Kimura 1980) $+\Gamma$ model, with a gamma shape parameter of 0.15 for $28 \mathrm{~S}$ and 0.34 for ITS-1. Pairwise genetic distances were calculated according to the optimised model, using the pairwise deletion option of gap-handling. A neighbour-joining (NJ) analysis was performed using the selected parameters and with the complete deletion option of gap-handling, assessing nodal support through 1000 bootstrap samples. Using 1000 replicates of Tree Bisection and Reconnection branch swapping, a maximum parsimony (MP) search was carried out making use of all sites.

Because of too little overlap, the ITS and $28 \mathrm{~S}$ sequences of Ligophorus leporinus (Zhang et Ji, 1981) (EF152321 and DQ537380 - Wu et al. 2007) and the 28S fragment ascribed to L. mugilinus (Hargis, 1955) (AF131710 - Mollaret et al. 2000; but see Blasco-Costa et al. 2012) were omitted from the analyses. The same applies for the $28 \mathrm{~S}$ sequence of $L$. vanbenedenii (Parona et Perugia, 1890) published by Wu et al. (2006) (DQ157655) as it was suggested to stem from another genus than Ligophorus (see Blasco-Costa et al. 2012, Sarabeev and Desdevises 2014). Hence, the analysis was chiefly based on the sequence dataset of Blasco-Costa et al. (2012) (GenBank accession Nos. JN996801JN996869), stemming from the Mediterranean and Black Sea representatives of Ligophorus, and our own data from Brazil. Trees were rooted using the lineages of Ligophorus that appeared as basal in the trees of Blasco-Costa et al. (2012).

In addition, other specimens (6 of L. saladensis and 4 of L. uruguayense) were stained with Gomori's trichrome, mounted in Histochoice (Amresco, Solon, OH, USA) and imaged using a Carl Zeiss LSM780 confocal fluorescence microscope and a PL APO $63 \times 1.4$ oil immersion lens. Three-dimensional (3D) stacks were acquired with a typical voxel size of $100 \times 100 \times 500 \mathrm{~nm}$ (XYZ). The sample was excited using the $488 \mathrm{~nm}$ line of an Argon Laser. Spectral emission was analysed using the internal GaAsP multianode detector and a $490 \mathrm{~nm}$ to $694 \mathrm{~nm}$ window $(8.9 \mathrm{~nm}$ subchannel window size). Spectral separation was used and the stain was separated from the general autofluorescence of the sample using the Linear Unmixing algorithm (weighted unmixing). After offline dye separation from autofluorescence, 3D stacks were further visualised using Imaris 7.4.2 software (Bitplane) and segmented with the isosurface option (with preliminary local background estimation). Finally, for the purpose of clarity, in some acquisitions, unwanted signal (remains of unseparated autofluorescence) was cut-off using the cut function of Imaris. The cut surface has no 3D texture.

\section{RESULTS}

Seventeen out of thirty (57\%) fingerlings of Mugil liza were infected with both Ligophorus uruguayense and L. saladensis. The overall mean abundance was 6.7 specimens of both species per individual fish.

\section{Phase contrast microscopy}

It was possible to distinguish two morphological groups identified as Ligophorus saladensis, based on the description provided by Marcotegui and Martorelli (2009), and 


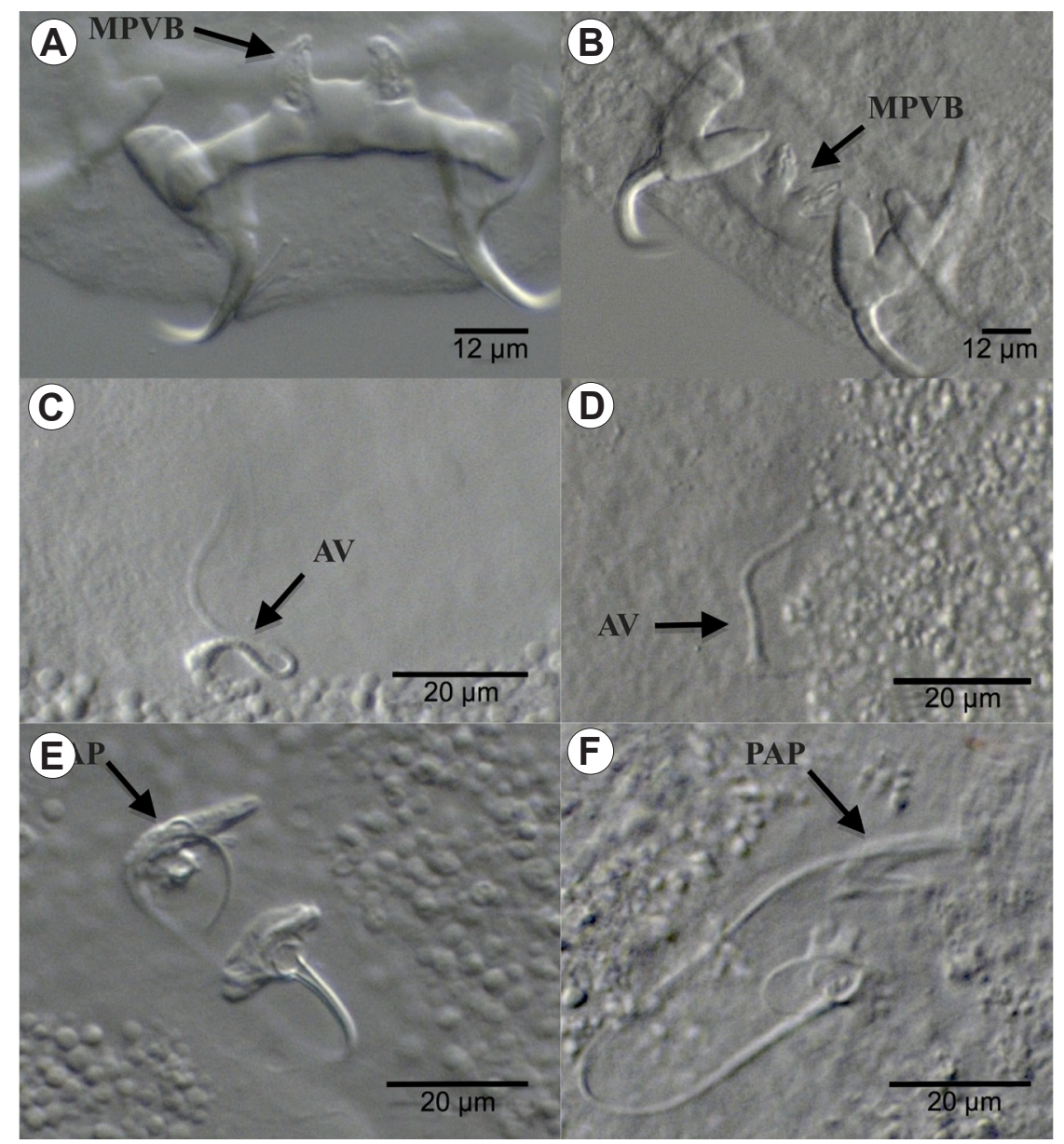

Fig. 2. Light micrographs of hard parts of specimen 1 (A, C, E) and specimen 2 (B, D, F) collected from Mugil liza Valenciennes in southern Brazil. A, B - ventral bar; C, D - sclerotised vagina; E, F - male copulatory complex. Abbreviations: AV - annulated vagina; MPVB - median process of the ventral bar; PAP - penis accessory piece.

L. uruguayense, which was described by Failla Siquier and Ostrowski de Núñez (2009), based on the shape of the median process of the ventral bar and the accessory piece of the MCC (Fig. 1).

However, the specimens studied revealed variation in other diagnostic features ascribed either to L. saladensis or L. uruguayense, such as the presence of transverse annulations at the distal end of the vaginal tube and a thick process at the distal end of the inner root of the ventral anchors (Fig. 2). Specimen 1 (Fig. 2A,C,E) possesses a ventral bar with a wide median-squared process characteristic for $L$. saladensis, but also an annulated vagina (a feature assigned to $L$. uruguayense). Moreover, the accessory piece displays an intermediate shape between the two species. Such condition was clearly caused by the position in which the structure was observed on the slide. Specimen 2 (Fig. 2B,D,F) exhibits the characteristic shape of the ventral bar, accessory piece and vagina for $L$. uruguayense, but lacks the remarkable thick process at the distal end of the inner root of the ventral anchor, which is allegedly characteristic to this species.

\section{Molecular characterisation}

A fragment spanning partial $18 \mathrm{~S}$ rDNA, the entire ITS$1 \mathrm{rDNA}$ spacer and the beginning of the 5.8S rRNA gene was sequenced from a specimen of L. uruguayense (722 bp; deposited in GenBank under accession No. KF442626) and from four specimens of L. saladensis (714 bp in view of indels as compared to L. uruguayense; KF442627). Their uncorrected pairwise genetic distance was 3\% (without indels and calculated over ITS-1 only, the other sequence portions being identical). From the 28S rRNA gene, $971 \mathrm{bp}$ were sequenced for $L$. saladensis (six specimens, yielding two haplotypes: KF442628, KF442629). For the two specimens belonging to $L$. uruguayense sequenced, the same $28 \mathrm{~S}$ rDNA fragment (KF442630) amounted to $970 \mathrm{bp}$ in view of an indel. The two species differed in 9 or 10 substitutions (equaling around $1 \%$ of uncorrected genetic distance, not counting the indel).

In the ITS-1 MP tree, $L$. saladensis formed a clade together with $L$. mediterraneus Sarabeev, Balbuena et Euzet, 2005 , with a bootstrap support of $86 \%$. In the $28 \mathrm{~S}$ MP tree, L. mediterraneus was nested within L. saladensis and clustered with one of its haplotypes with a bootstrap support of 68\% (not shown in Fig. 3). Based on ITS-1 as well as on $28 \mathrm{~S}$ rDNA sequences, the two species under study clustered firmly with L. mediterraneus (Fig. 3). Although relationships within this clade were poorly resolved, L. saladensis seems a sister species to L. mediterraneus in the $28 \mathrm{~S}$ tree. Only one or two substitutions (i.e. maximum 

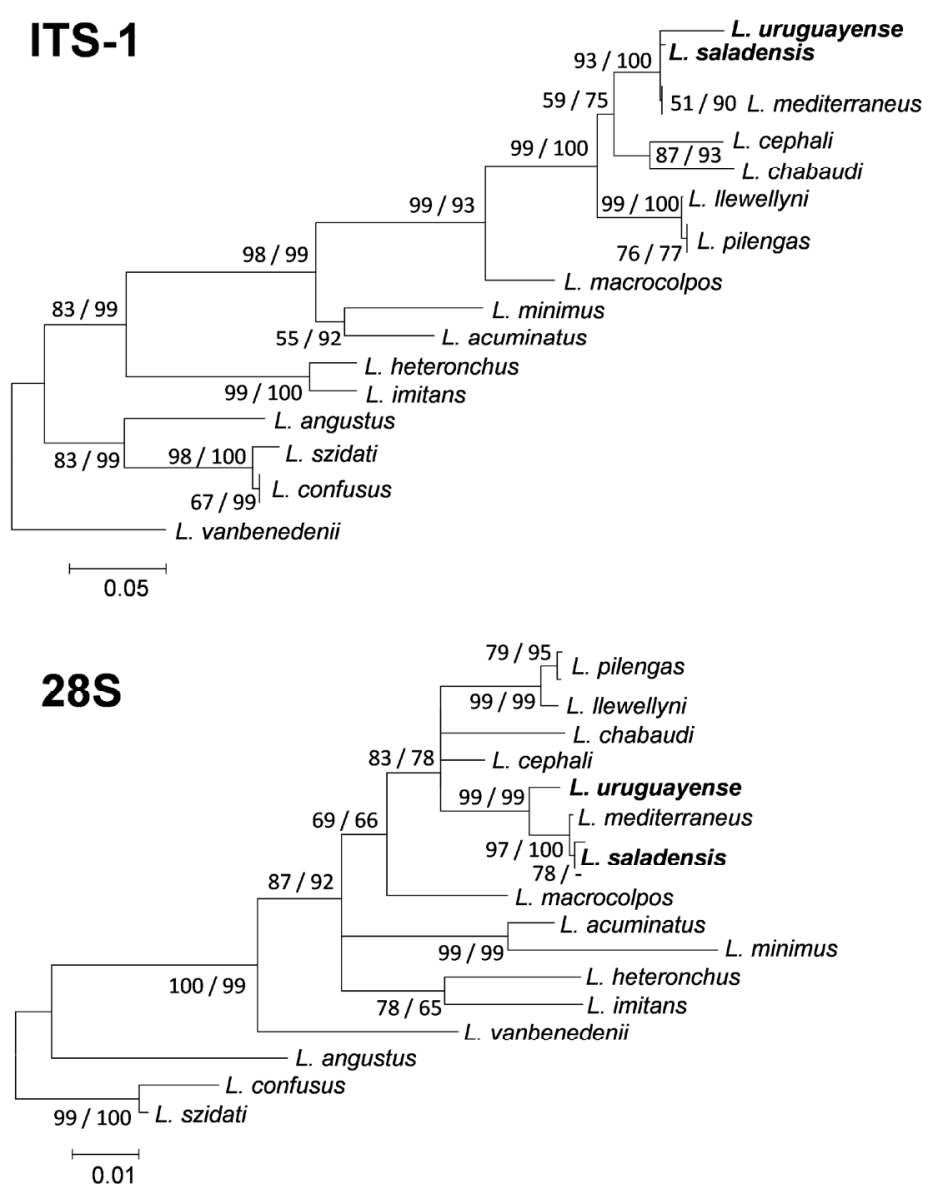

Fig. 3. Neighbour-joining phylogram constructed for the ITS-1 (above, 734 bp) and 28S (below, 18 unique haplotypes, 929 bp) rDNA sequences of Ligophorus Euzet et Suriano, 1977. The scale bar indicates the number of substitutions per site. The focal taxa of the present study are shown in bold. Statistical support for each node is shown: neighbour-joining bootstrap/maximum parsimony bootstrap. Nodes that did not receive a bootstrap support of at least 65 in at least one of both analyses are collapsed, creating a polytomy. A clade not recovered in a particular analysis is indicated by '-'.

$0.2 \%$ corrected pairwise genetic distance) were observed between the partial 28S rRNA gene sequences for these two species (Table 1). Their ITS-1 sequences only differed by $0.4-0.6 \%$. This distance is smaller than that between either of them and L. uruguayense (Table 1).

\section{Confocal fluorescence microscopy}

Imaging of species of Ligophorus under a confocal fluorescence microscope is depicted in Fig. 4. This provided accurate data on the morphology of the ventral bar and penis accessory piece for the two species.

The ventral bar of L. saladensis (Fig. 4C) is characterised by one median-squared process with an anteriorly extended membraneous protuberance on both sides. The protuberances and the median process are united to form a U-shaped anterior median part of the ventral bar. In contrast, the ventral bar of L. uruguayense (Fig. 4D) possesses one ventral, median $\mathrm{V}$-shaped process with an enlarged membraneous protuberance on both sides. The protuberances with the median process form a V-shaped anterior median part of the ventral bar.

As for the penis accessory piece, the upper lobe in L. saladensis is cylindrical, with two slightly spine-like protrusions where the accessory piece curves; these may serve in guiding the penis (Fig. 4E). The lower lobe is smaller than the upper lobe, with the distal tip turned away from the upper lobe. In L. uruguayense, this structure was shown to be quite similar to its original description (Fig. 4F); its upper lobe is cylindrical, convex and longer than the lower lobe, which, in turn, is straight and proximally united to the upper lobe.

\section{Morphometrics}

Comparative morphometric data obtained in the present study for L. uruguayense and L. saladensis and from their original descriptions are shown in Table 2. Similarly as observed by Marcotegui and Martorelli (2009), L. saladensis also presented greater VBAP, PAPL and LWL than L. uruguayense in the present study. The upper ranges for some characters of L. uruguayense reported in its original description (e.g. body width, VDIOR, DAA, DAB, DDIOR, VBL, dorsal bar length, PL and LWL) were lower than those found in the present study. Additionally, the ranges for some characters of $L$. saladensis found in the present study were outside the upper and lower ranges reported in its original description (e.g. body width, dorsal bar length and VAD). 


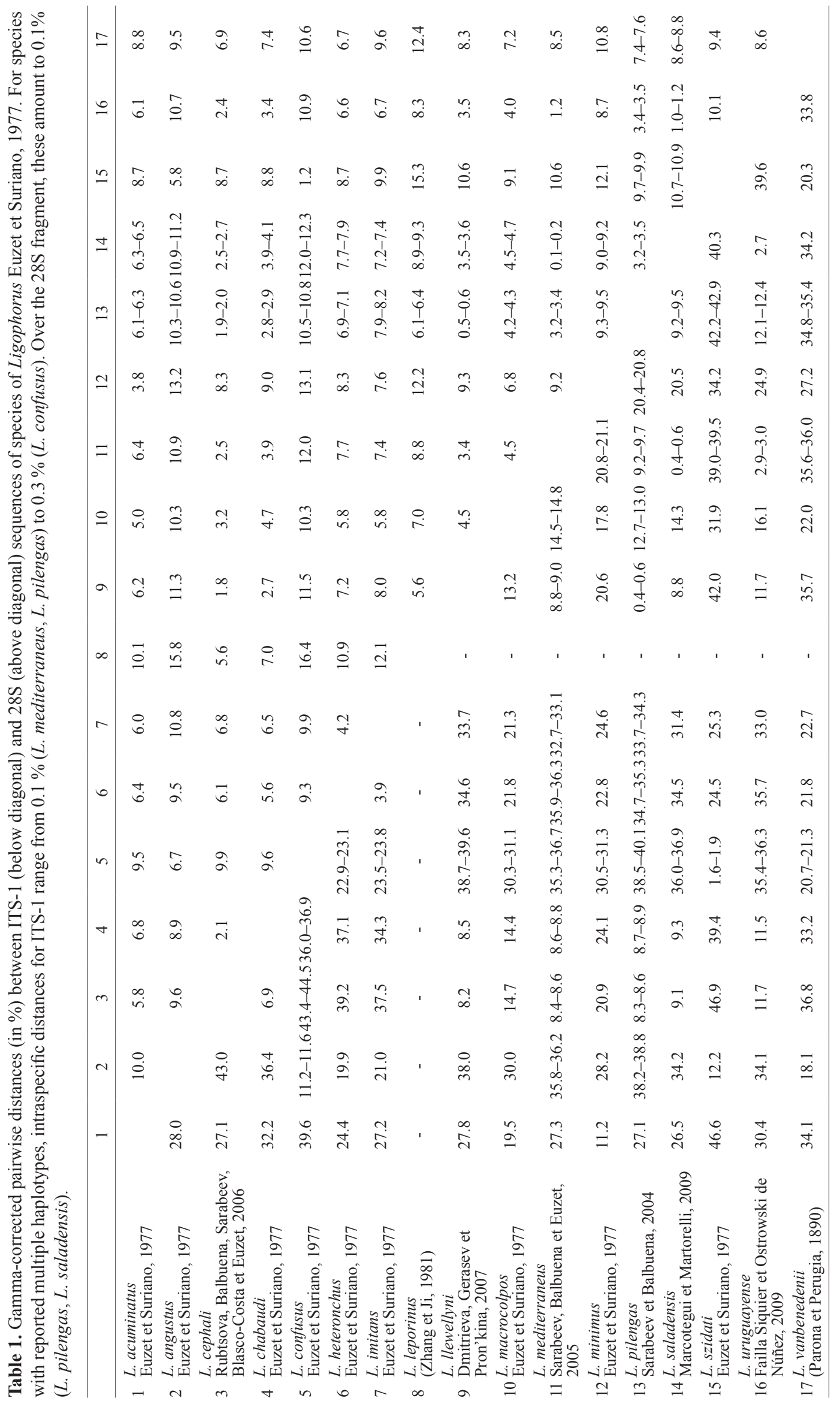




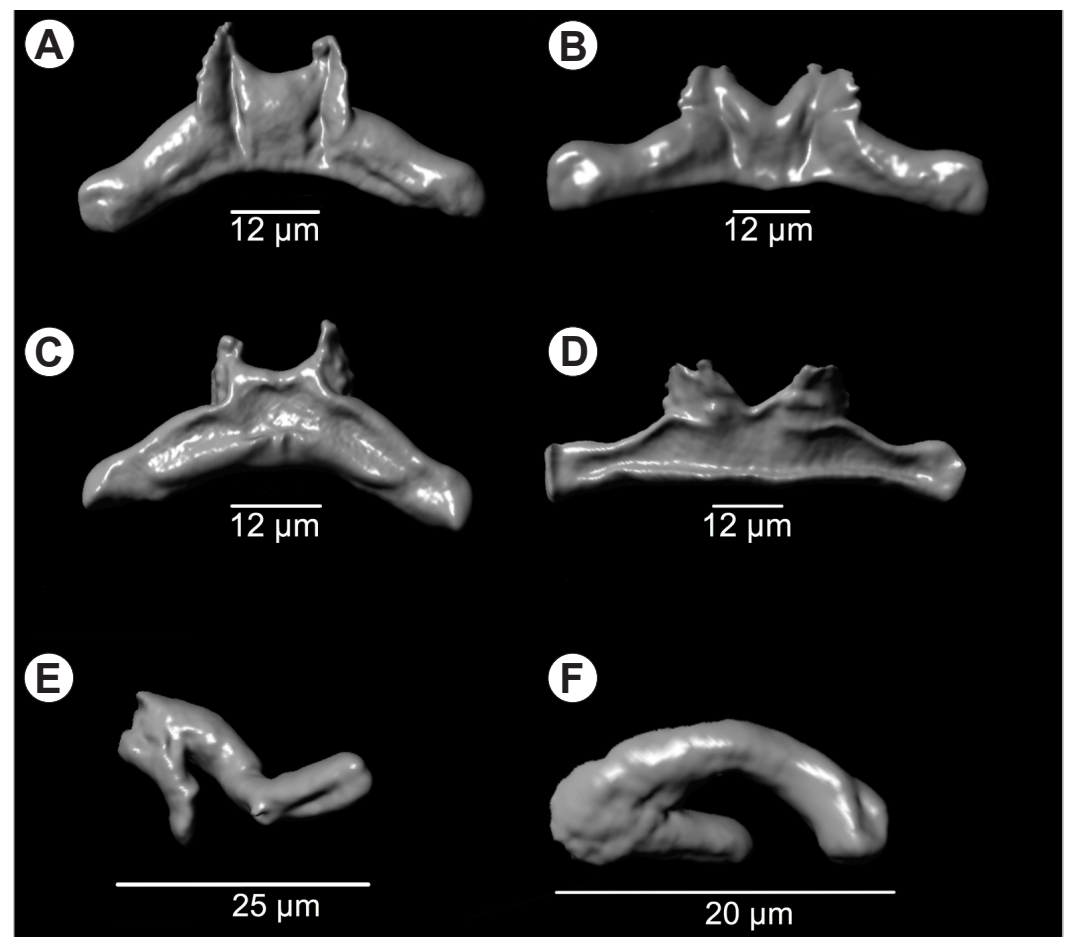

Fig. 4. Three-dimensional Imaris reconstruction of hard parts of Ligophorus saladensis Marcotegui et Martorelli, 2009 (A, C, E) and Ligophorus uruguayense Failla Siquier et Ostrowski de Núñez, 2009 (B, D, F) both collected from Mugil liza Valenciennes in southern Brazil. A, B - transverse bar, dorsal; C, D - transverse bar, ventral; E, F - penis accessory piece.

\section{DISCUSSION}

Since the erection of Ligophorus by Euzet and Suriano (1977), the number of species in the genus has increased to approximately 50. Nevertheless, according to BlascoCosta et al. (2012), this number can augment greatly as new hosts and localities are studied and, thus, the development of a reliable taxonomic framework based on morphological and molecular grounds is becoming increasingly needed (this was partially done by Sarabeev et al. 2013 and Sarabeev and Desdevises 2014). In the present study, we observed that scrutiny of the published diagnostic features under phase contrast microscopy does not succeed in unambiguously discriminating between $L$. saladensis and L. uruguayense.

Ligophorus uruguayense was described from M. liza (= M. platanus $)$ in Uruguay by Failla Siquier and Ostrowski de Núñez in 2009. It was distinguished from its congeners on the basis of the shape of the ventral bar and anchors, the accessory piece of the penis and the vaginal aperture, the host species and the geographical distribution. Later that same year, L. saladensis was described from the same fish host in Argentina by Marcotegui and Martorelli (2009). The authors differentiated their new species from other species of Ligophorus by the shape of the ventral bar and accessory piece and by morphometrics. Concerning its closely related species L. uruguayense, it was distinguished from the latter on the basis of the morphology of the accessory piece, the absence of transverse annulations at the distal end of the vaginal tube, as well as by the thick process at the distal end of the inner root of the ventral anchors. Additionally, L. saladensis was distinguished from
L. uruguayense by having a greater distance between the membraneous anterior processes (protuberances) of the ventral bar, and a greater total length and lower lobe length of the accessory piece.

The accessory pieces of the MCC (Fig. 4E,F) of L. saladensis and L. uruguayense can be sometimes very similar to each other, depending on the position in which this structure is examined, because of its delicate form and small size. Besides, its total length cannot be easily studied due to large amounts of vitelline follicles covering it. Nevertheless, each species presents a unique shape for this structure: whereas in L. uruguayense it has a smaller total length, with a cylindrical, convex upper lobe and straight lower lobe (because of that, it can easily remind of a 'box glove'), in L. saladensis it is longer, with the distal tip of the lower lobe turning away from the upper lobe.

Sarabeev et al. (2013) attributed a different morphology to the penis accessory piece of $L$. saladensis: according to them, it has a main lobe and a secondary one, the latter possessing a trident format, with three unequal branches. While the upper one extends beyond the distal end of the main lobe and is two times longer than the lower one, the medial branch has the smallest size. However, we did not identify such configuration in our analysed specimens. In addition, examination of one of the paratypes of $L$. saladensis (MLP-He 5935) deposited in the Collection of Invertebrates of the Museo de la Plata in Argentina revealed a similar morphology of the median process of the ventral bar and the accessory piece of the penis when compared to the presently studied specimens. Other characteristics 
Table 2. Comparison of morphometric characters (in micrometers, expressed as range with mean in parentheses) of Ligophorus uruguayense Failla Siquier et Ostroswki de Núñez, 2009 and Ligophorus saladensis Marcotegui et Martorelli, 2009 from present and previous studies.

\begin{tabular}{|c|c|c|c|c|}
\hline & \multicolumn{2}{|c|}{ Ligophorus uruguayense } & \multicolumn{2}{|c|}{ Ligophorus saladensis } \\
\hline & Present study & $\begin{array}{c}\text { Failla Siquier and } \\
\text { Ostrowski de Núñez (2009) }\end{array}$ & Present study & Marcotegui and Martorelli (2009) \\
\hline Body length & $513-742(652)$ & $614-1000$ & $597-731(650)$ & $523-798(532)$ \\
\hline Body width & $145-242(186)$ & $71-227$ & $185-244(220)$ & $82-120(132)$ \\
\hline \multicolumn{5}{|l|}{ Ventral anchor } \\
\hline VAA & $35-39(37)$ & $28-42$ & $28-36(30)$ & $29-38(34)$ \\
\hline VAB & $26-29(28)$ & $20-31$ & $22-27(24)$ & $21-33(26)$ \\
\hline VAC & $8-12(10)$ & $7-14$ & $9-13(11)$ & $10-15(12)$ \\
\hline VAD & $16(12-19)$ & $8-20$ & $13-15(14)$ & $16-23(17)$ \\
\hline VAE & $7-11(8)$ & $6-11$ & $8-9(8)$ & $9-12(9)$ \\
\hline VDIOR & $15-19(17)$ & $10-16$ & $13-22(18)$ & - \\
\hline \multicolumn{5}{|l|}{ Dorsal anchor } \\
\hline DAA & $32-54(39)$ & $28-46$ & $24-36(28)$ & $31-37(33)$ \\
\hline $\mathrm{DAB}$ & $23-42(28)$ & $19-33$ & $21-26(23)$ & $24-28(26)$ \\
\hline DAC & $8-16(11)$ & $6-15$ & $6-9(8)$ & $7-10(8)$ \\
\hline DAD & $14-24(18)$ & $7-22$ & $12-13(13)$ & $10-16(15)$ \\
\hline DAE & $7-13(9)$ & $6-12$ & $8-9(8)$ & $6-11(8)$ \\
\hline DDIOR & $13-29(19)$ & $8-16$ & $15-18(17)$ & - \\
\hline Hook total length & $9-11(10)$ & $8-13$ & $10-12(11)$ & $11-15(12)$ \\
\hline \multicolumn{5}{|l|}{ Ventral bar } \\
\hline VBL & $48-62(55)$ & $33-56$ & $50-55(53)$ & $53-62(58)$ \\
\hline VBAP & $4-9(6)$ & $5-9$ & $9-10(10)$ & $6-10(8)$ \\
\hline Dorsal bar length & $48-61(53)$ & $31-55$ & $47-55(50)$ & $31-44(37)$ \\
\hline \multicolumn{5}{|c|}{ Male copulatory complex } \\
\hline PAPL & $15-23(20)$ & $15-20$ & $23-28(25)$ & $19-28(21)$ \\
\hline PL & $104-121(110)$ & $87-115$ & $90-96(92)$ & $87-117(105)$ \\
\hline LWL & $8-18(11)$ & $8-12$ & $13-16(14)$ & $12-14(13)$ \\
\hline DBULL & $2-4(3)$ & $2-4$ & $3-4(3)$ & - \\
\hline Vagina length & 65 & $12-71$ & $59-63(61)$ & $55-65(57)$ \\
\hline
\end{tabular}

DAA - dorsal anchor total length; DAB - dorsal anchor main part length; DAC - dorsal anchor outer root length; DAD - dorsal anchor inner root length; DAE - dorsal anchor point length; DBULL - distance between upper and lower lobe of penis accessory piece; DDIOR - distance between inner and outer root of dorsal anchors; LWL - penis accessory piece lower lobe length; PAPL - penis accessory piece total length; PL - total length of penis; VAA - ventral anchor total length; VAB - ventral anchor main part length; VAC - ventral anchor outer root length; VAD - ventral anchor inner root length; VAE - ventral anchor point length; VBAP - distance between membranous anterior processes of ventral bar; VBL - ventral bar length; VDIOR - distance between inner and outer root of ventral anchors.

pointed out to be diagnostic ones by the original describers could not be observed.

Besides the shape of the accessory piece, Marcotegui and Martorelli (2009) also differentiated L. saladensis from $L$. uruguayense based on the absence of transverse annulations at the distal end of the vaginal tube and a thick process at the distal end of the inner root of the ventral anchors. However, in the presently studied specimens, neither the first nor the second character was found to be reliable for distinguishing one species from the other. Transverse annulations in the vagina were always present in both species, whilst the presence of a process in the inner root of the ventral anchors was highly variable for both of them (sometimes also present in L. saladensis). Furthermore, Sarabeev et al. (2013) also commented on the presence of transverse annulations at the distal end of the vaginal tube reported for L. uruguayense and concluded that this feature corresponds, to a muscular or fibrous sheath surrounding the sclerotised vaginal tube, being similar to that of other Ligophorus species.

With regard to the surprisingly wider ranges in some haptoral morphometrics of L. uruguayense signaled by
Sarabeev et al. (2013) (e.g. VAC, VAD, DAC and DAD) (but we found narrower ranges for these characters), they were still wider than those found for other Ligophorus species, including L. saladensis (see Table 2). The metrical differences found in the ranges between our observations and the original descriptions for both species should be considered as intraspecific ones or even a result of the different procedure applied (in the present study the measurements were taken from specimens fixed in $95 \%$ ethanol whereas they were taken from both fixed and live specimens and from heat-fixed specimens preserved in $10 \%$ formalin, respectively, in the original descriptions of L. uruguayense and L. saladensis).

Whereas the genetic divergence between L. saladensis and L. uruguayense is low as compared to most distances observed between representatives of Ligophorus (Table 1), comparable genetic distances between closely related species of Ligophorus exist (see also Blasco-Costa et al. 2012). Examples include L. llewellyni Dmitrieva, Gerasev et Pronkina, 2007 vs L. pilengas Sarabeev et Balbuena, 2004, and L. confusus Euzet et Suriano, 1977 vs L. szidati Euzet et Suriano, 1977. For example, despite their limited 
divergence, which may suggest L. llewellyni and L. pilengas are conspecific, Blasco-Costa et al. (2012) argued that the unambiguous morphological distinction between these species confirms species delineation. Similarly, considering the observed genetic and phenotypic differences between L. saladensis and L. uruguayense, our results support the validity of these species.

However, inter- and intraspecific distances seem to converge for the $28 \mathrm{~S}$ rDNA fragment for L. saladensis and L. mediterraneus, with interspecific phylogenetic relationships poorly resolved. Despite their morphological similarity (both species have a bilobed accessory piece of the MCC, with the lower lobe smaller than the upper, and a ventral bar with a median process; see also Dmitrieva et al. 2009a), the fact that they do not share haplotypes leads us to the suggestion that L. saladensis, L. mediterraneus and L. uruguayense belong to a complex of closely related species.

This situation is comparable for the host fishes: Mugil cephalus Linnaeus, the host of L. mediterraneus, probably belongs to the same species complex as M. liza. Both species are, despite their low genetic divergence, considered valid (Durand et al. 2012, Whitfield et al. 2012). The presence of separate but closely related parasite species on these closely related hosts corroborates the suggestion of El Hafidi et al. (2013) that species of Ligophorus can be used as a marker for taxonomy and evolution of mullet species.

To date, records for both L. uruguayense and L. saladensis are limited to the southwestern Atlantic. Whereas the former species was reported in Laguna de Rocha, Uruguay, the latter was later reported in Samborombón Bay, between Punta Piedras and Punta Rasa (Failla Siquier and Ostrowski de Núñez 2009, Marcotegui and Martorelli 2009).

Interestingly, although Samborombón Bay is very close to the Uruguayan coast, the authors reported only the presence of L. saladensis and no specimen of L. uruguayense was found. Furthermore, they suggested that the occurence of parasites could be related to host body size. However, this idea is not supported by the present study since our fish sample consisted of specimens with a body size of no more than 4 centimetres, which were parasitised by both parasite species.

It seems that because of the great latitudinal range of M. liza along the Atlantic coast, this host fish may face a wide spectrum of abiotic conditions. We can assume that speciation of species of Ligophorus is a result. This hypothesis was also supported by Pahor-Filho et al. (2012). Furthermore, we believe that future integrative studies similar to the present one should be carried out for other species of Ligophorus reported for M. liza in order to better comprehend the diversity and diversification of these fish parasites.

Blasco-Costa et al. (2012) suggest that both within-host duplication and host-switching contributed to the diversification of Ligophorus, whereas Sarabeev and Desdevises (2014) stress the importance of host-switching. The observed limited divergence between two species of Ligophorus, that share the same host raises the question as to which parasite speciation modes have occurred in this genus. To formally reconstruct a diversification scenario for the representatives of Ligophorus and to check for potential co-speciation events, co-phylogenetic analyses of this parasite and its mullet hosts are recommended.

Acknowledgments. The authors thank Coordination for Improvement of Higher Education Personnel (CAPES) for financial support to N.C. Marchiori and to J. Pereira Jr. (Project No. 23038.005284/2011-60) and the National Council for Scientific and Technological Development (CNPq; project No. $300753 / 2012-8$ ). Obtaining sequence newly produced during this work was financed by the Intitut de Recherche pour le Développement and obtained through the technical facilities of the "Centre Méditerraéen de l'Environnement et de la Biodiversité" (CeMEB). This is publication ISEM 2015-016 S. M.P.M. Vanhove was supported by a $\mathrm{PhD}$ fellowship of the Research Foundation - Flanders (FWO-Vlaanderen) and currently by the Czech Science Foundation (project no. P505/12/G112 - European Centre of Ichthyoparasitology).

\section{REFERENCES}

Abdallah V.D., Azevedo R.K., Luque J.L. 2009: Four new species of Ligophorus (Monogenea: Dactylogyridae) parasitic on Mugil liza (Actinopterygii: Mugilidae) from Guandu River, southeastern Brazil. J. Parasitol. 95: 855-864.

Blasco-Costa I., Míguez-Lozano R., Sarabeev V., BalBUENA J.A. 2012: Molecular phylogeny of species of Ligophorus (Monogenea: Dactylogyridae) and their affinities within the Dactylogyridae. Parasitol. Int. 61: 619-627.

Dmitrieva E.V., Gerasev P.I., Merella P., Pugachev O.N. 2009a: Redescription of Ligophorus mediterraneus Sarabeev, Balbuena and Euzet, 2005 (Monogenea: Ancyrocephalidae) with some methodological notes. Syst. Parasitol. 73: 95-105.

Dmitrieva E.V., Gerasev P.I., Merella P., Pugachev O.N. 2009b: Redescription of Ligophorus cephali Rubtsova, Balbuena, Sarabeev, Blasco-Costa and Euzet, 2006 and L. chabaudi Euzet and Suriano, 1977 (Monogenea: Ancyrocephalidae), with notes on the functional morphology of the copulatory organ. Syst. Parasitol. 73: 175-191.

Durand J.D., Shen K.N., Chen W.J., Jamandre B.W., Blel H., Diop K., Nirchio M., Garcia de León F.K., Whitfield A.K., Chang C.W., Borsa P. 2012: Systematics of the grey mullets (Teleostei: Mugiliformes: Mugilidae): molecular phylogenetic evidence challenges two centuries of morphology-based taxonomy. Mol. Phylogenet. Evol. 64: 73-92.

El Hafidi F., Berrada Rkhami O., de Buron I., Durand J.D., PARISElle A. 2013: Ligophorus species (Monogenea: Ancyrocephalidae) from Mugil cephalus (Teleostei: Mugilidae) off Morocco with the description of a new species and remarks about the use of Ligophorus spp. as biological markers of host populations. Folia Parasitol. 60: 433-440.

Euzet L., Suriano D.M. 1977: Ligophorus n. g. (Monogenea, Ancyrocephalidae) parasite des Mugilidae (Téléostéens) en Mediterranée. Bull. Mus. Natl. Hist. Nat. Zool. 472: 799-821.

Failla Siquier G.F., Ostrowski de Núñez M. 2009: Ligophorus uruguayense sp. nov. (Monogenea, Ancyrocephalidae), a gill parasite from Mugil platanus (Mugiliformes, Mugilidae) in Uruguay. Acta Parasitol. 54: 95-102.

Fraga E., Schneider H., Nirchio M., Santa-Brigida E., RoDRIGUES-Filho L.F., SAMPAIO I. 2007: Molecular phylogenetic analyses of mullets (Mugilidae, Mugiliformes) based on two mitochondrial genes. J. Appl. Ichthyol. 23: 598-604. 
Humason, G.L. 1979: Animal Tissue Techniques. Fourth Edition. W.H. Freeman and Company, San Francisco, 661 pp.

Kimura M. 1980: A simple method for estimating evolutionary rate of base substitutions through comparative studies of nucleotide sequences. J. Mol. Evol. 16: 111-120.

Lockyer A.E., Olson P.D., Littlewood D.T.J. 2003: Utility of complete large and small subunit rRNA genes in resolving the phylogeny of the Neodermata (Platyhelminthes): implications and a review of the cercomer theory. Biol. J. Linn. Soc. 78: $155-171$.

Littlewood D.T.J., Curini-Galletti M., Herniou E.A. 2000 The interrelationships of Proseriata (Platyhelminthes: Seriata) tested with molecules and morphology. Mol. Phylogenet. Evol. 16: $449-466$

Malmberg G. 1957: [On the occurrence of Gyrodactylus in Swedish fishes.] Skrifter utgivna av Södra Sveriges Fiskeriföreningen 1956: 19-76. (In Swedish.)

Marcotegui P.S., Martorelli S.R. 2009: Ligophorus saladensis n. sp. (Monogenea: Ancyrocephalidae) from Mugil platanus Günther in Samborombón Bay, Argentina. Syst. Parasitol. 74: 41-47.

Mollaret I., Jamieson B.G.M., Justine J.L. 2000: Phylogeny of the Monopisthocotylea and Polyopisthocotylea (Platyhelminthes) inferred from 28S rDNA sequences. Int. J. Parasitol. 30: 171-185.

Pahor-Filho E., Miranda-Filho K.C., Pereira J. Jr. 2012: Parasitology of juvenile mullet (Mugil liza) and effect of formaldehyde on parasites and host. Aquaculture 354-355: 111-116.
Sarabeev V., Desdevises Y. 2014: Phylogeny of the Atlantic and Pacific species of Ligophorus (Monogenea: Dactylogyridae): morphology vs. molecules. Parasitol. Int. 63: 9-20.

Sarabeev V., Rubtsova N., Yang T., Balbuena J.A. 2013: Taxonomic revision of the Atlantic and Pacific species of Ligophorus Euzet and Suriano, 1977 (Monogenea: Dactylogyridae) from mullets (Teleostei: Mugilidae) with proposal of a new genus and description of four new species. Vestn. Zool. 2013, Suppl. 28: $1-112$.

Tamura K., Stecher G., Peterson D., Filipski A., Kumar S. 2013: MEGA6: Molecular Evolutionary Genetics Analysis Version 6.0. Mol. Biol. Evol. 30: 2725-2729.

Thompson J.D., Higgins D.G., Gibson T.J. 1994: CLUSTAL W: improving the sensitivity of progressive multiple sequence alignment through sequence weighting, position-specific gap penalties and weight matrix choice. Nucl. Acids Res. 22: 4673-4680.

Whitfield A.K., Panfili J., Durand J.D. 2012: A global review of the cosmopolitan flathead mullet Mugil cephalus Linnaeus, 1758 (Teleostei: Mugilidae), with emphasis on the biology, genetics, ecology and fisheries aspects of this apparent species complex. Rev. Fish Biol. Fisher. 22: 641-681.

Wu X.Y., Zhu X.Q., Xie M.Q., Li A.X. 2006: The radiation of Haliotrema (Monogenea: Dactylogyridae: Ancyrocephalinae) molecular evidence and explanation inferred from LSU rDNA sequences. Parasitology 132: 659-668.

Wu X.Y., Zhu X.Q., XIE M.Q., Li A.X. 2007: The evaluation for generic-level monophyly of Ancyrocephalinae (Monogenea, Dactylogyridae) using ribosomal DNA sequence data. Mol. Phylogenet. Evol. 44: 530-544.

Cite this article as: Marchiori N.C., Pariselle A., Pereira Jr. J., Agnèse J.-F., Durand J.-D., Vanhove M.P.M. 2015: A comparative study of Ligophorus uruguayense and L. saladensis (Monogenea: Ancyrocephalidae) from Mugil liza (Teleostei: Mugilidae) in southern Brazil. Folia Parasitol. 62: 024. 